LfD Baden-Württemberg: 30 Jahre Volkszählungsurteil - aktueller denn je

„Das Volkszählungsurteil des Bundesverfassungsgerichts ist aktueller denn je“, erklärte der Landesbeauftragte für den Datenschutz Baden-Württemberg, Jörg Klingbeil, anlässlich des 30. Jahrestags der am 15. Dezember 1983 ergangenen Entscheidung. „Das Grundrecht auf informationelle Selbstbestimmung muss weiterhin der Maßstab für den Datenschutz der Zukunft sein, nicht nur wegen der persönlichen Freiheitsrechte des Einzelnen, sondern wegen des Zusammenhalts unserer Gesellschaft überhaupt.

Die damaligen Feststellungen des Gerichts gelten unverändert fort: Wer nicht weiß, ob sein Verhalten beobachtet und registriert wird, wird sich nicht mehr unbefangen bewegen und kann seine Freiheitsrechte nicht mehr uneingeschränkt ausüben. Gemessen an den heutigen - selbstverursachten oder fremdbestimmten - Gefährdungen der Privatsphäre mag der damalige Anlass Volkszählung als ein kleiner Stein des Anstoßes erscheinen. Umso wichtiger ist es, die Grundprinzipien des Volkszählungsurteils in die heutige Zeit zu übersetzen und zugleich wetterfest für die Herausforderungen der Zukunft zu machen." Ein wesentlicher Handlungsstrang sei dabei - so Jörg Klingbeil - die europäische Integration, die eine gemeinsame Antwort auf die globalen, vor allem die technischen Rahmenbedingungen - z.B. des Internet - erfordere. Hierfür seien nun mit der Europäischen Datenschutzreform erste Eckpfeiler erkennbar, die die Kernelemente des deutschen Datenschutzrechts auch zum Maßstab für Europa machen könnten. Dafür sei aber erforderlich, dass die Bundesregierung aus dem Bremserhäuschen herauskomme und das Vorhaben nachhaltig vorantreibe.

Eine europäische Datenschutzgrundverordnung könne mit einem darin verankerten Marktortprinzip dafür sorgen, dass europäische Spielregeln auch für außereuropäische Anbieter gelten, zum Beispiel soweit es um die Information der Nutzer über die Weitergabe von Daten in Drittstaaten gehe.

Damit könnten allerdings die Aktivitäten ausländischer Geheimdienste noch nicht wirksam eingefangen werden, hierfür seien völkerrechtliche Vereinbarungen unabdingbar. Das sei der zweite politische Handlungsauftrag, der aktuell aus der Rechtsprechung des Bundesverfassungsgerichts angesichts der NSA-Spähaffäre erwachse. Der Landesbeauftragte erinnerte in diesem Zusammenhang an zwei weitere wegweisende Entscheidungen des Gerichts: „Das Bundesverfassungsgericht hat 2008 neben das Grundrecht auf informationelle Selbstbestimmung das Grundrecht auf Vertraulichkeit und Integrität informationstechnischer Systeme gestellt und damit die Rahmenbedingungen des digitalen Zeitalters aufgegriffen. Dieses Grundrecht muss endlich mit Leben erfüllt werden, zum Beispiel indem der Staat die Infrastruktur für eine sichere Kommunikation wesentlich mehr fördert und ausbaut. Ferner hat das Gericht 2010 in seiner Entscheidung zur Vorratsdatenspeicherung das Verbot einer totalen Überwachung und Registrierung der Freiheitswahrnehmung der Bürger als Element der verfassungsrechtlichen Identität der Bundesrepublik Deutschland bezeichnet, für dessen Wahrung sich unser Staat in europäischen und internationalen Zusammenhängen einsetzen müsse. Es ist höchste Zeit, dass die Regierung diesen Verfassungsauftrag umsetzt und sich der Totalüberwachung durch ausländische Geheimdienste aktiv entgegenstemmt. Dazu gehört aber auch, dass die eigene Auslandsaufklärung kritisch überprüft und zurückgefahren wird. Genauso wenig, wie wir es akzeptieren können, wenn die NSA ihre weltweite Schnüffelei damit verteidigt, dass, nur' Ausländer - also wir alle - be- troffen seien, können wir es hinnehmen, dass der Bundesnachrichtendienst Ausländer wie Rechtlos behandelt. Erforderlich ist daher eine völkerrechtlich wirksame Beschränkung für Geheimdienstaktivitäten. Hier haben sich durch die Enthüllungen Edwards Snowdens Sümpfe aufgetan, die es trockenzulegen gilt!"

\section{Cryptovision: Ein Beispiel einer Produktoffensive gegen PRISM und Co.}

Kaum ein Tag vergeht derzeit ohne neue Meldungen über die Schnüffelaktivitäten der NSA. Die Nachfrage nach Verschlüsselungstechnik ist daher in den vergangenen Monaten deutlich angestiegen. Deutsche Kryptologie-Hersteller profitieren besonders davon, denn die deutsche Kryptologie hat international einen erstklassigen Ruf. Im Gegensatz zu den USA, Frankreich, Israel und einigen anderen Ländern, in denen Krypto-Lösungen produziert werden, hat sich der Staat in Deutschland bisher nie in die Arbeit der Krypto-Hersteller eingemischt. Deutsche Kryptologie-Produkte stehen daher nicht im Verdacht, Hintertüren eingebaut zu haben.

Der Gelsenkirchener Krypto-Hersteller cryptovision hat sich schon vor über zehn Jahren auf die Bedrohung durch Geheimdienste eingestellt. Die E-Mail-Verschlüsselungslösung cv act s/ mail, die unter anderem von der Bundeswehr eingesetzt wird, fügt eigenständige Sicherheitsmodule mit Smartcard-Anbindung in die E-Mail-Programme Outlook und Notes ein. Selbst wenn das jeweilige E-Mail-Programm manipuliert sein sollte, ist die Verschlüsselung nicht beeinträchtig. Der hohe Sicherheitsstandard von cv act s/mail wird unter anderem durch eine Einsatzempfehlung des BSI und eine Zertifizierung der NATO bestätigt.

Auf den NSA-Skandal reagiert cryptovison nun mit zwei neuen Produkt-Releases. Da ein Unternehmen zur Verschlüsselung eine Public-Key-Infrastruktur (PKI) benötigt, gibt es derzeit eine beträchtliche Nachfrage nach dieser Technologie. cryptovisions vollmodulare PKI-Lösung CAmelot (jetzt in Version 2.0) wurde daher weiter ausgebaut. Dieses hochflexible Produkt trägt der Tatsache Rechnung, dass sich Public-Key-Infrastrukturen oft deutlich voneinander unterscheiden und im Laufe des Betriebs geändert oder erweitert werden müssen. Dank der vollmodularen Architektur sind auch außergewöhnliche Architekturen und nachträgliche Anpassungen einfach möglich.

Auch die universelle Smartcard-Middleware cv act sc/interface erfreut sich derzeit großer Beliebtheit. Viele Unternehmen haben erkannt, dass nur mit Smartcards eine NSA-sichere Verschlüsselung gewährleistet ist. cv act sc/interface unterstützt alle relevanten Kartentypen auf allen relevanten Betriebssystemen über alle relevanten Schnittstellen. Mit einem neuen Release (Version 6.0) wird diese Flexibilität nun noch erweitert. Damit ist cv act sc/interface ideal geeignet, um in nahezu jeder Umgebung Smartcards für die Verschlüsselung einzusetzen.

\section{Hardware-Verschlüsselungslösung für Hochsicherheit direkt am Arbeitsplatz}

Das am 28.11.2013 von secunet (http://www.secunet.com/) vorgestellte Layer 2 Verschlüsselungssystem rundet die SINA Layer 2 Produktfamilie mit einer Verschlüsselungsleistung von $50 \mathrm{MBit} / \mathrm{s}$ auf 